Ann. Sci. forest., 1971, 28 (4), 403-423.

\title{
INFLUENCE DE L'ENGORGEMENT DE PRINTEMPS ET DE LA SÉCHERESSE D'ÉTÉ SUR LE COMPORTEMENT DE JEUNES PLANTS D'ÉPICÉA
}

\author{
G. LÉVY \\ avec la collaboration technique de M. GuiLLEmETte \\ Station de Recherches sur les Sols forestiers et la Fertilisation, \\ Centre national de Recherches forestières, I.N.R.A., \\ 54 - Champenoux
}

\section{RÉSUMÉ}

Cette expérience concernait de jeunes semis d'Épicea (Picea abies) repiqués dans des pots de terre de $30 \mathrm{~cm}$ de hauteur situés dans une serre; elle s'est poursuivie pendant deux ans. Différents traitements ont été appliqués au printemps : $\mathrm{C}=$ sol maintenu à la capacité au champ, $\mathrm{F}, \mathrm{M}$ et $\mathrm{R}=$ nappe d'eau maintenue respectivement à $-7,-14$ et $-21 \mathrm{~cm}$. Ils ont été suivis de trois traitements d'été : $F^{\prime}=$ sécheresse forte, réhumectation environ une fois par mois, $M^{\prime}$ ' = sécheresse moyenne (réhumectation 2 fois plus fréquente), $C^{\prime}=$ sol maintenu à la capacité au champ; il y a donc 12 traitements complets.

Les résultats essentiels sont les suivants :

- la nappe la plus superficielle $(F)$ se traduit par la croissance en hauteur la plus faible sauf si elle est suivie d'un bon approvisionnement en eau pendant l'été (C'); elle provoque également l'enracinement le moins dense et le moins profond, quel que soit le traitement d'été; elle a aussi pour conséquence une très mauvaise nutrition azotée des plants.

- la nappe à profondeur intermédiaire $(\mathrm{M})$ constitue par contre l'éventualité la plus favorable de toutes pour la croissance en hauteur des plants et pour leur survie.

- les deux nappes les moins superficielles ( $M$ et $R$ ) sont plus efficaces, pour la croissance en hauteur, qu'un approvisionnement convenable en eau, mais sans nappe (C).

- ce n'est que parmi les plants à fort desséchement du sol l'été (F') que l'on enregistre un taux de mortalité élevé; seuls ceux qui ont subi le traitement de printemps (M) y échappent.

\section{I. - INTRODUCTION}

Le programme de recherches concernant les forêts sur sols à hydromorphie temporaire, qui intéresse d'importantes surfaces notamment dans le nord-est de la France, a pour objet essentiel de déterminer, pour chaque type de station hydromorphe, la ou les essences que l'on 
peut conseiller d'y introduire, ainsi que la manière éventuelle dont il est souhaitable d'intervenir sur les conditions édaphiques.

Aussi ce programme comporte-t-il des «projets » de recherches destinés, les uns à examiner les relations entre la croissance des arbres et les propriétés du sol, ce qui devrait permettre de prévoir la production dans chaque station donnée, les autres à étudier l'influence de divers procédés d'amélioration (fertilisation et surtout assainissement) sur le comportement de certaines essences (reprise, croissance). Les premiers sont réalisés par « enquêtes », il ne s'agit donc que d'une observation des situations existantes, dont il est impossible en l'absence de confirmation expérimentale de déduire des relations impliquant une causalité. Les seconds sont réalisés par expérimentation sur le terrain : les différences que l'on peut observer, si elles sont significatives, ont bien pour origine les différences de traitement; mais il est des problèmes qu'ils ne permettent pas de résoudre car, de même que dans le cas des " enquêtes", certains facteurs, et en particulier les flictuations de la nappe, sont aléatoires.

En fait, si l'on désire déterminer l'influence réelle de la nappe sur le comportement des plants, il est nécessaire de contrôler son niveau et ses fluctuations. Alors, on peut espérer expliquer les phénomènes observés dans la nature et leur donner une dimension bien plus générale. C'est pourquoi le programme sur les sols à hydromorphie temporaire comporte un troisième volet : les expérimentations sous serre. Plusieurs «projets » s'y rattachent. Pour certains d'entre eux, le facteur que l'on fait varier est la nappe : on teste différents niveaux d'afleurement ou diverses durées et périodicités de submersion sur la mortalité et la croissance des parties aériennes des plants, sans négliger les facteurs explicatifs que peuvent constituer la nutrition (analyses foliaires) ou surtout le développement du système radiculaire. D'autres projets ont pour objet de tester certains moyens de lutte autres que l'assainissement (fertilisation, mélange d'essences), le facteur « nappe » étant identique dans tous les traitements.

En réalité la réalisation de la plupart de ces projets n'a pas encore débuté. L'un par contre est pratiquement terminé : c'est de ce projet que nous rendons compte dans ce mémoire. Son but est de comparer différents niveaux d'affleurement de la nappe dans le sol, ainsi que de tester, pour chacun de ces niveaux, divers régimes de déssèchement pendant l'été : on reproduit donc dans une certaine mesure les conditions auxquelles les arbres sont soumis dans la nature sur les sols à pseudogley.

Après avoir décrit les méthodes utilisées, nous indiquerons les résultats relatifs à la croissance aérienne, au développement du système radiculaire, à la mortalité et à la nutrition. Puis nous tenterons, avec une synthèse des divers résultats, d'en déduire une explication de certains phénomènes observés dans la nature, principalement dans le domaine des jeunes plantations (en raison de la faible taille du matériel végétal utilisé).

\section{II. - MÉTHODES}

\section{1. - Matériel végétal}

L'Epicéa commun (Picea abies) est l'essence de reboisement la plus utilisée jusqu'à présent sur sols à pseudogley. La principale "enquête » sur les relations sol-production concerne cette essence à laquelle on a fait également appel dans les expériences d'assainissement où elle servira de référence pour les autres especes testées. C'est pourquoi nous avons utilisé l'Epicéa pour cette première expérience sous serre. 


\section{2. - Substrat et localisation}

Les semis 1-0 d'Épicéa ont été repiqués dans de grands pots de terre $(30 \mathrm{~cm}$ de hauteur, $30 \mathrm{~cm}$ de diamètre intérieur) à raison de 7 semis par pot.

On avait préalablement mis $4 \mathrm{~cm}$ de graviers dans le fond du pot, que l'on avait ensuite rempli à l'aide d'un horizon $\mathrm{A}_{2} \mathrm{~g}$ d'un sol à pseudogley prélevé en forêt d'Amance, les $5 \mathrm{~cm}$ supérieurs étant constitués par l'horizon $\mathrm{A}_{1}$ du même sol. Les analyses de sol sont reproduites dans le tableau 1 .

On peut constater que les teneurs en matière organique et en éléments nutritifs sont satisfaisantes, de même que le $\mathrm{pH}$ et le rapport $\mathrm{C} / \mathrm{N}$, et que la texture est limoneuse à limono-argileuse : le substrat utilisé est bien représentatif de l'horizon engorgé de nombreux sols à pseudogleys formés sur roche-mère argileuse recouverte de limons éoliens sur une épaisseur très souvent supérieure à $30 \mathrm{~cm}$.

Les pots destinés à recevoir une nappe sont placés dans 2 grands bacs métalliques de $4 \mathrm{~m}$ de longueur sur 1,20 m de largeur et $0,40 \mathrm{~m}$ de hauteur, à différentes distances du fond du bac (ils sont placés sur des supports plus ou moins hauts); les bacs sont remplis d'eau jusqu'à un certain niveau. Les autres pots sont placés sur une estrade à côté des bacs.

L'expérience est localisée sous une serre chauffée l'hiver de façon à éviter le gel et ventilée pendant les températures élevées d'été.

\section{3. - Traitements}

Chaque pot subit au cours d'une année 2 traitements successifs : celui de la phase de printemps (ayant commencé début mai la $1^{\text {re }}$ année, début mars la $2^{\text {e }}$ année) et celui de la phase d'été (qui commence mi-juin et se termine en octobre); puis, après les quelques mois d'hiver, pendant lesquels tous les pots sont alimentés normalement et de la même façon en eau (apport deux fois par semaine), les 2 traitements sont reproduits, pour chaque pot au cours de la $2^{\mathrm{e}}$ année de repiquage, identiques à ce qu'ils étaient l'année précédente.

Il y a quatre traitements principaux pendant la phase de printemps :

- F : eau affleurant à $-7 \mathrm{~cm}$ de la surface du sol

$-\mathrm{M}: \ll \quad » \quad \dot{\mathrm{a}}-14 \mathrm{~cm}$

$-\mathbf{R}: \ll \quad » \quad \dot{a}-21 \mathrm{~cm}$

- C : pots sans nappe, mais dont l'approvisionnement en eau est satisfaisant (apport deux fois par semaine).

Le niveau de la nappe est maintenu identique à lui-même pendant toute la phase de printemps. A la fin de cette phase, l'eau est retirée des bacs.

Trois traitements principaux sont appliqués pendant la phase d'été :

- $\mathrm{F}^{\prime}$ : fort dessèchement du sol, réhumectation quand le $\mathrm{pF}$ de rétention de l'eau par le sol atteint ou dépasse 4 à $-15 \mathrm{~cm}$ dans au moins la moitié des pots où l'on a mis des sondes en plâtre; celà correspond à une réhumectation d'environ une fois par mois.

- $\mathrm{M}^{\prime}$ : sécheresse moyenne, réhumectation avec une périodicité à peu près double de $\mathrm{F}^{\prime}$.

- $\mathrm{C}^{\prime}$ : réhumectation 2 fois par semaine, comme le traitement de printemps $\mathrm{C}$.

En associant 2 à 2 les traitements de printemps et ceux d'été, on aboutit à 12 modalités principales $\left(\mathrm{FF}^{\prime}, \mathrm{FM}^{\prime}\right.$, etc...). Il y a 6 pots par modalité, plus quelques pots destinés à être sacrifiés à la fin de la $1^{\text {re }}$ phase (ils n'ont subi que le traitement de printemps). Dans la mesure du possible, les pots ont été répartis au hasard.

Il y a en outre 2 modalités accessoires, qui ne comportent que 3 pots chacune :

- $\mathrm{SM}^{\prime}$ : qui correspond à une sécheresse moyenne pendant les 2 phases (traitement $\mathrm{M}^{\prime}$ appliqué en permanence)

$-\mathrm{MH}^{\prime}$ : dont les pots ont une nappe affleurant à $-14 \mathrm{~cm}$ pendant les 2 phases

$$
\text { 2.4. - Mesures }
$$

A la fin de chaque phase, donc 2 fois par an, on procède à un certain nombre de mesures :

- Croissance en hauteur : on mesure la pousse de tous les plants et on effectue les moyennes par pot et par modalité.

- Enracinement : on sacrifie un pot par modalité à la fin de chaque phase, du moins pour les modalités principales, puis on mesure, pour chacun des plants, la longueur maximale de l'enracine- 
TABLEAU 1 - TABle 1

Analyses du sol utilisé

Analysis of the soil used

\begin{tabular}{|c|c|c|c|c|c|c|c|c|c|c|c|c|c|c|}
\hline Horizon & $\begin{array}{c}\text { Mat. } \\
\text { org. } \\
\% \\
\text { (organic } \\
\text { matter) }\end{array}$ & $\begin{array}{l}\mathrm{N} \\
\%\end{array}$ & $\mathrm{C} / \mathrm{N}$ & $\mathrm{pH}$ & $\begin{array}{l}\mathrm{Ca} \\
\text { (1) }\end{array}$ & $\begin{array}{c}\mathrm{K} \\
(1)\end{array}$ & $\begin{array}{l}\mathrm{Mg} \\
\text { (1) }\end{array}$ & $\begin{array}{c}\mathrm{T} \\
(1)\end{array}$ & $\begin{array}{c}\mathrm{P}_{2} \mathrm{O}_{5} \\
\%\end{array}$ & $\begin{array}{c}\text { Argile } \\
\% \\
\text { (Clay) }\end{array}$ & $\begin{array}{c}\text { Limons } \\
\text { fins } \\
\% \\
\text { (fine silt) }\end{array}$ & $\begin{array}{c}\text { Limons } \\
\text { grossiers } \\
\% \\
\text { (coarse silt) }\end{array}$ & $\begin{array}{c}\text { Sables } \\
\text { fins } \\
\% \\
\text { (fine sand) }\end{array}$ & $\begin{array}{c}\text { Sables } \\
\text { grossiers } \\
\% \\
\text { (coarse sand) }\end{array}$ \\
\hline $\mathbf{A}_{1}$ & 4,9 & 0,20 & 14 & 5,4 & 6,2 & 0,43 & 0,98 & 12,8 & 0,19 & 19,1 & 38,5 & 25,5 & 4,1 & 4,0 \\
\hline $\mathrm{A}_{2} \mathrm{~g}$ & 2,1 & 0,11 & 11 & 4,8 & 2,3 & 0,11 & 0,36 & 9,2 & 0,13 & 21,2 & 41,7 & 27,7 & 3,3 & 2,7 \\
\hline
\end{tabular}

(1) en milliéquivalents pour $100 \mathrm{~g}$. 
ment ainsi que le nombre de racines à $-4 \mathrm{~cm},-6 \mathrm{~cm},-8 \mathrm{~cm}$, etc... Pour chacune de ces mesures on calcule la moyenne pour l'ensemble des plants du pot.

- Mortalité : on compte le nombre de plants morts dans chaque modalité.

- Nutrition : des analyses foliaires sont effectuées; celles qui correspondent à la fin de la phase de printemps ne seront interprétées que prudemment car la composition des aiguilles est loin d'être stable à cette époque.

\section{5. - Interprétation}

L'interprétation statistique des résultats a été effectuée par analyse de variance à deux facteurs contrôlés : on compare les traitements de printemps entre eux et les traitements d'été entre eux, chacun de ces deux groupes de traitement constituant un facteur contrôlé.

Les résultats dont nous allons rendre compte concernent les deux années ayant suivi le repiquage; les plants ont donc subi 4 phases successives ( 2 traitements de printemps et 2 d'été).

$$
\text { III. - RÉSULTATS }
$$

\section{1. - La croissance en hauteur}

Nous allons d'abord présenter les résultats concernant la croissance totale des plants au cours des deux années de repiquage, puis nous les comparerons aux diverses mesures partielles.

La comparaison des résultats relatifs aux diverses modalités ne tiendra pas compte des deux modalités accessoires $\left(\mathrm{SM}^{\prime}\right.$ et $\left.\mathrm{MH}^{\prime}\right)$; celles-ci seront commentées à part.

3.11. Croissance totale au cours des deux années (tableau 2).

TABLEAU 2 -- TABLE 2

Somme des 2 pousses annuelles (valeurs moyennes, en $\mathrm{cm}$ )

Sum of the two annual height increases (mean values, in $\mathrm{cm}$ )

\begin{tabular}{|c|c|c|c|c|c|}
\hline & $\mathrm{F}$ & M & $\mathrm{R}$ & C & $S$ \\
\hline$F^{\prime}$ & 11,2 & 14,8 & 12,7 & 12.2 & \\
\hline$M^{\prime}$ & 9,3 & 13,5 & 12,5 & 12,2 & $(14,3)$ \\
\hline $\mathrm{C}^{\prime}$ & 12,0 & 16,8 & 17,5 & 13,1 & \\
\hline $\mathrm{H}^{\prime}$ & & $(20,3)$ & & & \\
\hline
\end{tabular}

L'analyse de variance fournit les résultats suivants :

— inégalités significatives :

- $\quad$ à $1 \%: \mathrm{F}<\mathrm{M}, \mathrm{F}<\mathrm{R}$

- à $5 \%: \mathrm{C}<\mathrm{M}$

Les trois modalités $\mathrm{F}$ occupent les trois dernières positions, de peu pour $\mathrm{FC}^{\prime}$. D'autre part, les trois modalités $\mathrm{M}$ sont parmi les quatre meilleures; elles ont toutes trois une moyenne 
$\geqslant 13,5 \mathrm{~cm}$; de plus, si l'on ne considère que les deux traitement d'été les plus défavorables $\left(\mathrm{F}^{\prime}\right.$ et $\left.\mathrm{M}^{\prime}\right)$, on constate que les deux modalités $\mathrm{M}\left(\mathrm{MF}^{\prime}\right.$ et $\left.\mathrm{MM}^{\prime}\right)$ sont toutes deux supérieures aux modalités $\mathrm{R}$, lesquelles sont supérieures aux modalités $\mathrm{C}$; les deux modalités $\mathrm{F}\left(\mathrm{FM}^{\prime}\right.$ et $\mathrm{FF}^{\prime}$ ) ont les croissances les plus faibles de toutes.

La comparaison des trois traitements d'été fait apparaître les inégalités significatives suivantes :

$$
\mathrm{C}^{\prime}>\mathrm{M}^{\prime} \text { et } \mathrm{C}^{\prime}>\mathrm{F}^{\prime}
$$

D'autre part, parmi les trois modalités intéressant chacun des quatre traitements de printemps, c'est toujours celle subissant $C^{\prime}$ qui a la meilleure croissance (ainsi $R^{\prime}>R^{\prime}$ et $\mathrm{RM}^{\prime}, \mathrm{MC}^{\prime}>\mathrm{MF}^{\prime}$ et $\mathrm{MM}^{\prime}$, etc...)

\subsection{Croissance au cours des deux premières phases (printemps et été 1969).}

A la fin de la première phase (printemps 1969), les différences entre pousses moyennes sont assez faibles; ces pousses varient de $4,2 \mathrm{~cm}(\mathrm{~F})$ à $5,2 \mathrm{~cm}(\mathrm{R})$.

La pousse annuelle ne s'accroit que faiblement au cours de la deuxième phase (la période normale d'élongation est passée). La pousse totale 1969 varie de $4,2 \mathrm{~cm}$ pour FC' à $6,7 \mathrm{~cm}$ pour $\mathrm{RC}^{\prime}$. L'analyse de variance donne les résultats suivants :

— inégalités significatives :

- à $1 \%: \mathrm{F}<\mathrm{M}, \mathrm{F}<\mathrm{C}$

- à $5 \%: \mathrm{F}<\mathrm{R}$.

Par contre les traitements d'été ne sont pas significativement différents les uns des autres.

Les trois modalités $\mathrm{F}\left(\mathrm{FM}^{\prime}, \mathrm{FC}^{\prime}\right.$ et $\left.\mathrm{FF}^{\prime}\right)$ ont les trois moyennes de pousses les plus faibles, parmi les 12 (ou même 14) modalités. Ce résultat est en accord avec celui enregistré pour la croissance totale $1969+1970$.

\subsection{Croissance au cours des $3^{\mathrm{e}}$ et $4^{\mathrm{e}}$ phases (printemps et été 1970) (tableau 3).}

Nous n'indiquons pas séparément les résultats relatifs à chacune de ces deux phases pour la raison suivante : de même qu'au cours de la première année de repiquage (1969), la croissance en hauteur au cours de la phase d'été est faible par rapport à la pousse de printemps (celle-ci varie suivant les modalités de 4, 3 à $9,0 \mathrm{~cm}$ et la pousse totale 1970 de 5,5 à $11 \mathrm{~cm}$ ). Aussi est-il logique de constater que les résultats concernant la $3^{\mathrm{e}}$ phase seule et ceux relatifs à l'ensemble de l'année 1970 sont pratiquement identiques. On peut également supposer d'après la remarque précédente, que l'effet d'un traitement d'été ne se manifeste, sur la croissance en hauteur des plants, qu'au cours du printemps suivant; c'est d'ailleurs ce que l'on constate : ce n'est pratiquement qu'à partir de la $3^{\mathrm{e}}$ phase que le traitement d'été provoque une discrimination entre les croissances en hauteur des diverses modalités.

Cette même constatation amène ceci : la pousse 1969 ne dépendant pratiquement que du traitement de printemps, il sera plus logique de prendre en considération les résultats relatifs à la seule pousse 1970 que ceux qui concernent la pousse totale $1969+1970$.

Précisons tout d'abord que si la pousse totale n'est pas toujours égale à la somme des deux pousses annuelles c'est en raison des mortalités intervenues entre-temps. 
TABLEAU 3 - TABLE 3

Pousse 1970 (valeurs moyennes, en cm)

Height increase in 1970 (mean values, in cm)

\begin{tabular}{|c|c|c|c|c|c|}
\hline & $\mathrm{F}$ & $\mathrm{M}$ & $\mathrm{R}$ & $\mathrm{C}$ & $\mathrm{S}$ \\
\hline$F^{\prime}$ & 6,6 & 8,7 & 7,5 & 6,8 & \\
\hline$M^{\prime}$ & 5,5 & 8,1 & 6,8 & 6,0 & $(10,0)$ \\
\hline$C^{\prime}$ & 8,2 & 10,7 & 11,0 & 8,0 & \\
\hline $\mathrm{H}^{\prime}$ & & $(13,4)$ & & & \\
\hline
\end{tabular}

Les inégalités suivantes sont significatives :

- à $1 \%: \mathrm{F}<\mathrm{C}, \mathrm{F}<\mathrm{M}, \mathrm{R}<\mathrm{M}, \mathrm{M}^{\prime}<\mathrm{C}^{\prime}, \mathrm{F}^{\prime}<\mathrm{C}^{\prime}$

- à $5 \%: \mathrm{M}^{\prime}<\mathrm{F}^{\prime}$.

La majorité des constatations effectuées pour la pousse totale reste globalement valable pour la seule pousse 1970. Cependant, il existe certaines divergences. On peut faire les constatations suivantes en ce qui concerne la pousse 1970 :

- La comparaison des traitements de printemps montre que, pour un traitement d'été identique, $\mathrm{M}$ et $\mathrm{R}$ sont toujours plus favorables que $\mathrm{C}$ et $\mathrm{F}$. Comme pour la pousse totale, les trois modalités $\mathrm{M}$ ont une pousse parmi les meilleurs, $>8 \mathrm{~cm}$, mais, contrairement à elle, les trois modalités $\mathrm{F}$ ne sont plus les trois plus mauvaises de toutes, en particulier $F^{\prime}$. Si l'on ne considère que les deux traitements d'été les plus défavorables $\left(F^{\prime}\right.$ et $\left.M^{\prime}\right)$, le résultat diffère peu de celui qui concerne la pousse totale : les deux modalités $M\left(M^{\prime}\right.$ et $\mathrm{MM}^{\prime}$ ) sont toutes deux supérieures à toutes les autres, alors que les deux modalités $\mathrm{R}$ $\left(\mathrm{RF}^{\prime}\right.$ et $\left.\mathrm{RM}^{\prime}\right)$ sont supérieures aux deux modalités $\mathrm{F}\left(\mathrm{FF}^{\prime}\right.$ et $\left.\mathrm{FM}^{\prime}\right)$.

- Si l'on compare les trois traitements d'été, l'effet favorable de $\mathrm{C}^{\prime}$ apparaît encore plus nettement que pour la pousse totale. L'ensemble des modalités $C^{\prime}$ a une croissance significativement supérieure à celle des modalités $M^{\prime}$ et $F^{\prime}$. D'autre part, parmi les trois modalités intéressant chacun des quatre traitements de printemps, c'est toujours celle subissant $C^{\prime}$ qui a la meilleure croissance. De plus, et celà ne s'observait pas pour la pousse totale, toutes les modalités $\mathrm{C}^{\prime}$, même $\mathrm{FC}^{\prime}$, ont une pousse 1970 assez favorable, en tous cas $\geqslant 8 \mathrm{~cm}$. Ces résultats sont à mettre en parallèle avec le fait suivant : les plants soumis à $C^{\prime}$ ont débourré sensiblement plus tôt que les autres en 1970 ( $3^{\mathrm{e}}$ phase).

\subsection{Conclusions partielles.}

Nous allons tenter de tirer les enseignements résultants de l'ensemble des mesures de croissance en hauteur, particulièrement de celles qui concernent la pousse 1970 (pour la raison indiquée précédemment). Ce sont les suivantes :

- Lorsqu'il y a nappe dans le sol (niveau constant pendant toute la phase de printemps), il existe un seuil très net entre les nappes affleurant à -14 et $-7 \mathrm{~cm}$, celà si 1'on considère un même traitement d'été quel qu'il soit : la pousse dans ce dernier cas $(-7 \mathrm{~cm})$ est nettement la plus faible de toutes. 
- Pour un même traitement d'été, une nappe même relativement superficielle, mais pas trop ( $-14 \mathrm{ou}-21 \mathrm{~cm}$ dans le cas de très jeunes plants d'Epicéa tout au moins) est préférable à une alimentation convenable en eau (arrosage 2 fois par semaine) mais sans présence de nappe.

- Quel que soit le traitement d'été (sécheresse ou non), les plants ayant subi pendant le printemps une nappe affleurant à $-14 \mathrm{~cm}$ ont une croissance en hauteur parmi les meilleures.

- Quand il existe une certaine sécheresse d'été, qu'elle soit moyenne ou forte, cette même nappe (affleurant à $-14 \mathrm{~cm}$ ) constitue l'éventualité la plus favorable de toutes; elle est notamment préférable à une nappe un peu plus profonde $(-21 \mathrm{~cm})$. Par contre ce dernier cas est préférable à une nappe très superficielle $(-7 \mathrm{~cm})$.

- La pousse est relativement forte dans tous les pots qui ont reçu une alimentation en eau satisfaisante pendant l'été (bien plus élevée que dans ceux qui ont subi une sécheresse d'été, à traitement de printemps équivalent). Les pots de la modalité $\mathrm{FC}^{\prime}$, qui avaient eu une pousse relativement très faible au cours de l'année 1969, ont eu une croissance en hauteur au contraire très convenable (par rapport aux autres modalités) en 1970, car l'influence du traitement d'été ne peut se manifester, comme nous l'avons constaté précédemment, que pendant le printemps suivant. Un engorgement superficiel dans un pseudogley n'est donc défavorable à la croissance des plants d'Epicéa que s'il est accompagné d'une certaine sécheresse pendant l'été.

— En ce qui concerne les 2 modalités « accessoires", on peut faire les remarques suivantes :

- $\mathrm{MH}^{\prime}$ est la modalité fournissant toujours la pousse la plus forte. Celà confirme le fait qu'une nappe, à condition qu'elle ne soit pas trop superficielle, est préférable (pour la croissance en hauteur) à un apport convenable d'eau mais sans présence de nappe; ce phénomène était apparu précédemment lors de la phase de printemps, nous voyons maintenant qu'il reste valable pour la phase d'été.

- la croissance en hauteur pour la modalité SM' est bonne : un certain desséchement du sol pendant le printemps n'est pas défavorable (mais cette modalité ne correspond évidemment pas à des conditions de pseudogley).

\section{2. - L'enracinement}

Certaines anomalies pouvant apparaître dans la comparaison de l'enracinement à la fin de deux phases successives sont la conséquence du fait que ce ne sont pas les mêmes plants qui sont examinés dans les deux cas.

\subsection{Fin de la $1^{\text {re }}$ phase (printemps 1969).}

Les longueurs maximales d'enracinement (moyenne de la profondeur atteinte par la racine la plus profonde de chacun des plants du pot sacrifié) sont les suivantes, en $\mathrm{cm}$ :

$$
\mathrm{C}=16 . \quad \mathrm{M} \text { et } \mathrm{R}=12 . \quad \mathrm{F}=6 .
$$


Donc les racines s'arrêtent 1 ou $2 \mathrm{~cm}$ au-dessus de la nappe. (Signalons que lors du repiquage, pratiquement toutes les racines s'arrêtaient vers $-8 \mathrm{~cm}$.)

Nous avons par ailleurs évalué la densité d'enracinement en comptant le nombre de racines de chacun des plants des pots sacrifiés tous les deux $\mathrm{cm}$ à partir de $-4 \mathrm{~cm}$ et en effectuant les moyennes par modalité, à chaque profondeur.

La densité d'enracinement pour $\mathrm{F}$ est nettement la plus faible de toutes, à chaque profondeur (tableau 2) (Inégalités significatives à $1 \%$ ). Jusqu'à $-12 \mathrm{~cm}$, elle est peu différente pour les trois autres traitements.

\section{TABLEAU 4 - TABLE 4}

Nombres moyens de racines par plant à la fin de la $1{ }^{\mathrm{re}}$ phase (printemps 1969)

Mean quantity of roots for a seedling at the end of the first phasis (spring 1969)

\begin{tabular}{|c|c|c|c|c|c|c|c|c|}
\hline $\begin{array}{r}\text { Profondeur }(\mathrm{cm}) \\
\text { (deepness) }\end{array}$ & \multirow[b]{2}{*}{-4} & \multirow[b]{2}{*}{-6} & \multirow[b]{2}{*}{-8} & \multirow[b]{2}{*}{-10} & \multirow[b]{2}{*}{-12} & \multirow[b]{2}{*}{-14} & \multirow[b]{2}{*}{-16} & \multirow[b]{2}{*}{-18} \\
\hline $\begin{array}{ll}\begin{array}{l}\text { Modalité } \\
\text { (treatment) }\end{array} & (\mathrm{cm})\end{array}$ & & & & & & & & \\
\hline$F$ & 2 & 1 & & & & & & \\
\hline M & 7 & 7 & 5 & 2 & 1 & & & \\
\hline $\mathrm{R}$ & 6 & 7 & 5 & 2 & 1 & & & \\
\hline C & 8 & 9 & 6 & 3 & 2 & 2 & 2 & 1 \\
\hline
\end{tabular}

\subsection{Fin de la $2^{\mathrm{e}}$ phase (été 1969).}

Contrairement à ce qui se passe pour la pousse aérienne, la longueur et la densité des racines se sont très nettement accrues pendant l'été, du moins pour les trois traitements comportant une nappe ( $\mathrm{F}, \mathrm{M}$ et $\mathrm{R})$.

Les longueurs maximales d'enracinement sont indiquées dans le tableau 5.

TABLEAU 5 - TABLE 5

Longueurs maximales d'enracinement à la fin de la $2^{\mathrm{e}}$ phase (en $\mathrm{cm}$ )

Maximum lenght of the rooting at the end of the second phasis (in $\mathrm{cm}$ )

\begin{tabular}{|c|c|c|c|c|c|}
\hline & $\mathrm{F}$ & $\mathbf{M}$ & $\mathrm{R}$ & $\mathrm{C}$ & S \\
\hline$F^{\prime}$ & 14 & 24 & 14 & 16 & \\
\hline$M^{\prime}$ & 12 & 21 & 25 & 19 & (24) \\
\hline$C^{\prime}$ & 10 & 18 & 20 & 19 & \\
\hline $\mathrm{H}^{\prime}$ & & (12) & & & \\
\hline
\end{tabular}


Les inégalités suivantes sont significatives :

- à $1 \%: \mathrm{F}<\mathrm{M}$

- à $5 \%: \mathrm{F}<\mathrm{C}, \mathrm{F}<\mathrm{R}$.

Pour tous ces traitements $\mathrm{F}$ ou $\mathrm{M}$, les racines dépassent nettement la profondeur où affleurait la nappe de printemps.

Les trois modalités F restent cependant celles dont l'enracinement est le moins profond, mais, phénomène nouveau, les modalités $\mathrm{C}$ n'ont plus la longueur d'enracinement la plus grande.

Quant aux densités d'enracinement (tableau 6), on peut remarquer que, par rapport à la fin de la $1^{\text {ere }}$ phase, elles sont nettement plus fortes (parfois du double ou plus) à toute profondeur pour $\mathrm{F}, \mathrm{M}$ et $\mathrm{R}$. Jusqu'à $-12 \mathrm{~cm}$ inclus, aucune différence entre traitement de printemps ou d'été n'est significative. Sous cette profondeur, les traitement $F$ ont une densité d'enracinement (nulle) significativement inférieure à celle des trois autres traitements de printemps.

TABLEAU 6 - TABLE 6

Nombre moyen de racines par plant à la fin de la $2^{\mathrm{e}}$ phase (été 1969)

Mean quantity of roots for a seedling at the end of the second phasis (summer 1969)

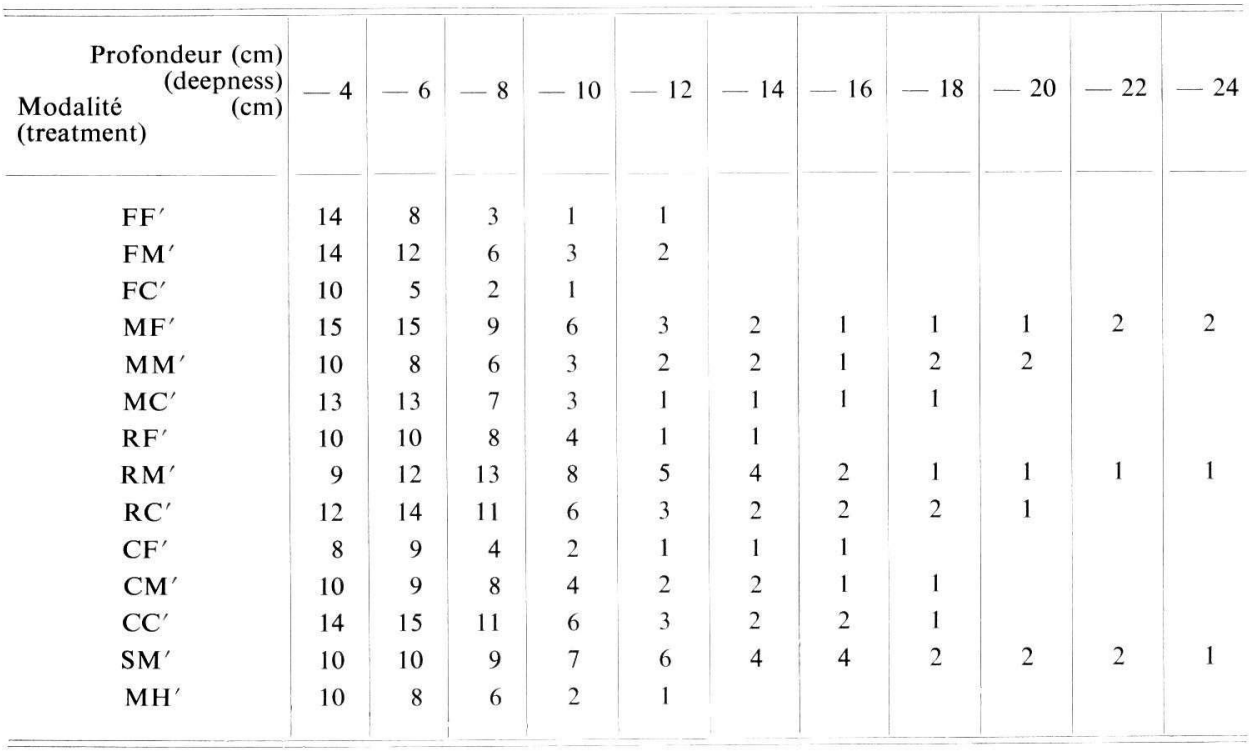

\subsection{Fin de la $3^{\mathrm{e}}$ phase (printemps 1970)}

Les longueurs maximales d'enracinement pour les diverses modalités sont indiquées dans le tableau 7 . 
TABLEAU 7 - TABLE 7

Longueurs maximales d'enracinement à la fin de la $3^{\mathrm{e}}$ phase (en $\mathrm{cm}$ )

Maximum lenght of the rooting at the end of the third phasis (in $\mathrm{cm}$ )

\begin{tabular}{c|c|c|c|c}
\hline \hline & F & M & R & C \\
\hline$F^{\prime}$ & 6 & 14 & 17 & 20 \\
$M^{\prime}$ & 7 & 13 & 18 & 17 \\
$\mathrm{C}^{\prime}$ & 7 & 14 & 19 & 16 \\
\hline
\end{tabular}

On peut constater que les racines sont ramenées au niveau d'affleurement de la nappe pour les modalités $\mathrm{F}$ et $\mathrm{M}$ et un peu au dessus de la nappe pour $\mathrm{R}$. La profondeur d'enracinement pour le traitement $\mathrm{C}$ est voisine de celle correspondant à $\mathbf{R}$.

Même les racines dont la croissance n'était pas bloquée par la présence d'une nappe (traitement C) n'ont pratiquement pas poussé depuis la fin de la $2^{\mathrm{e}}$ phase.

En ce qui concerne la densité de l'enracinement aux différentes profondeurs (tableau 8), celle des modalités $\mathrm{C}$ ou $\mathrm{F}$ ne s'est pas améliorée par rapport à la fin de la phase précédente, bien au contraire pour certaines d'entre elles; c'est pour $\mathrm{FF}^{\prime}$ qu'elle est nettement la plus faible. Par contre elle a augmenté en profondeur ( $\mathrm{de}-8 \mathrm{~cm}$ à la nappe) pour les modalités $\mathrm{M}$ et $\mathrm{R}$ : elle est parfois du double (les comparaisons entre phases différentes sont délicates, car il ne s'agit pas des mêmes plants : on ne peut en tirer que des tendances).

\section{TABLEAU 8 - TABLE 8}

Nombres moyens de racines par plant à la fin de la $3^{\mathrm{e}}$ phase (printemps 1970)

Mean quantity of roots for a seedling at the end of the third phasis (spring 1970)

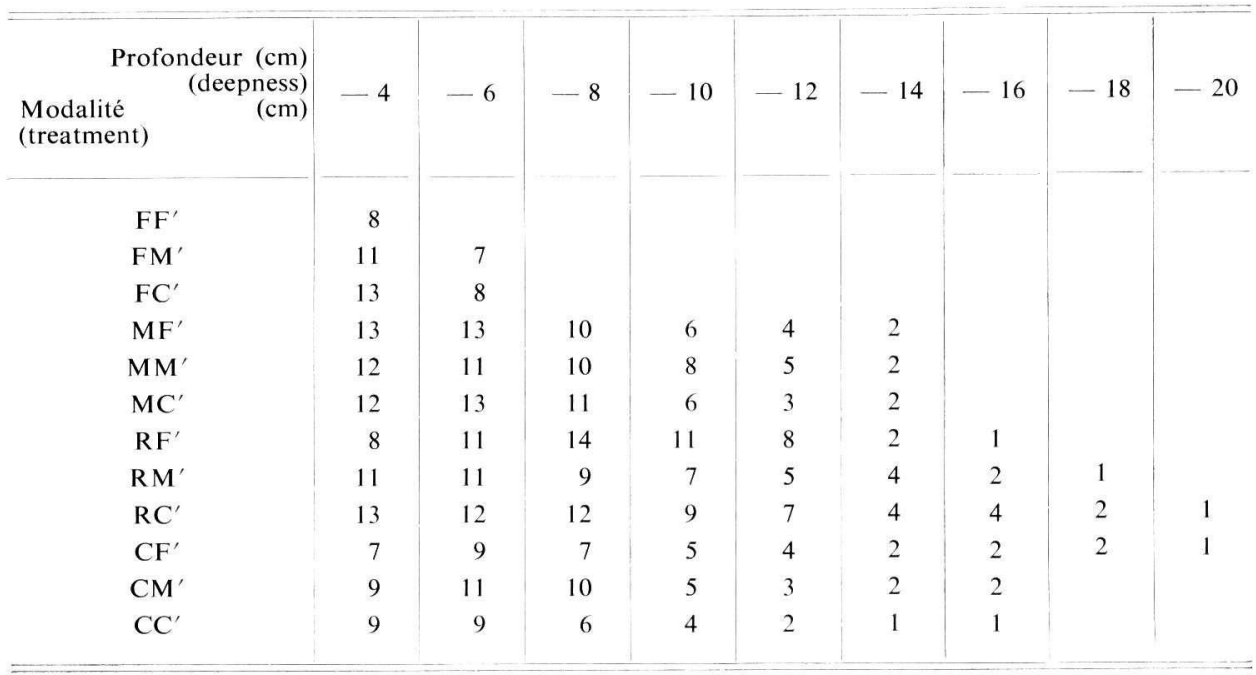


Il n'y a, à aucune profondeur, de différence très significative de densité d'enracinement entre modalités non engorgés à ce niveau.

\subsection{L'enracinement à la fin des deux années.}

Les longueurs maximales d'enracinement sont indiquées dans le tableau 9.

TABLEAU 9 - TABLE 9

Longueurs maximales d'enracinement à la fin des 2 années (en $\mathrm{cm}$ )

Maximum lenght of the rooting at the end of the two years (in $\mathrm{cm}$ )

\begin{tabular}{l|c|c|c|c|c}
\hline \hline & F & M & R & C & S \\
\hline $\mathrm{F}^{\prime}$ & & & & & \\
$\mathrm{M}^{\prime}$ & 14 & 27 & 27 & 27 & \\
$\mathrm{C}^{\prime}$ & 23 & 24 & 27 & 27 & (27) \\
& 15 & 23 & 27 & 27 & \\
\hline \hline
\end{tabular}

Les inégalités suivantes sont significatives :

- à $1 \%: \mathrm{F}<\mathrm{R}, \mathrm{F}<\mathrm{C}$

- à $5 \%: \mathrm{F}<\mathrm{M}$.

Remarquons que la croissance en longueur des racines a été très nette au cours de la $4^{\mathrm{e}}$ phase.

Les plants atteignent pratiquement la partie inférieure des pots dans sept modalités sur douze. Les trois modalités dont l'enracinement est nettement le moins profond sont les modalités $\mathrm{F}$ (surtout $\mathrm{FC}^{\prime}$ et $\mathrm{FF}^{\prime}$ ). Par contre pour toutes les modalités $\mathrm{C}$ et $\mathrm{R}$, les plants atteignent le fond du pot; ce sont elles d'ailleurs dont l'enracinement était déjà le plus profond à la fin de la $3^{\mathrm{e}}$ phase.

La profondeur de l'enracinement dépend donc essentiellement du traitement de printemps.

En ce qui concerne la densité de l'enracinement (tableau 10), on peut faire les constatations suivantes :

- Les différences entre traitement de printemps ou d'été ne sont plus significatives jusqu'à $-12 \mathrm{~cm}$. A partir de $-14 \mathrm{~cm}$, les traitement $\mathrm{F}$ sont significativement inférieurs aux trois autres traitements de printemps. C'est pour $\mathrm{FF}^{\prime}$ que la densité d'enracinement est pratiquement partout la moins bonne, même à -4 et $-6 \mathrm{~cm} ; \mathrm{FF}^{\prime}$ avait déjà la densité d'enracinement nettement la plus faible à la fin de la $3^{\mathrm{e}}$ phase.

- Les autres traitements de printemps, par contre, ne s'avèrent pas différents si on les examine globalement.

- A $-4 \mathrm{~cm}$, pour chaque traitement de printemps, c'est le traitement d'été $\mathrm{F}^{\prime}$ qui se traduit par la densité d'enracinement la plus faible. Par contre à partir de $-8 \mathrm{~cm}, \mathrm{~F}^{\prime}$ donne des résultats globalement peu différents de ceux de $\mathbf{M}^{\prime}$. 
TABLEAU 10 - TABLE 10

Nombres moyens de racines par plant

à la fin de la $4^{\mathrm{e}}$ phase (été 1970)

Mean quantity of roots for a seedling at the end of the fourth phasis (summer 1970)

\begin{tabular}{|c|c|c|c|c|c|c|c|c|c|c|c|c|}
\hline $\begin{array}{r}\begin{array}{r}\text { Profondeur } \\
\text { (deepness) } \\
(\mathrm{cm})\end{array} \\
\text { Modalité } \\
\text { (treatment) }\end{array}$ & -4 & -6 & -8 & -10 & -12 & -14 & -16 & -18 & -20 & -22 & -24 & -26 \\
\hline $\mathrm{FF}^{\prime}$ & 12 & 11 & 10 & 11 & 6 & 4 & & & & & & \\
\hline $\mathrm{FM}^{\prime}$ & 18 & 16 & 13 & 12 & 8 & 7 & 3 & 2 & 2 & 1 & & \\
\hline $\mathrm{FC}^{\prime}$ & 26 & 16 & 11 & 11 & 7 & 4 & & & & & & \\
\hline $\mathrm{MF}^{\prime}$ & 15 & 19 & 23 & 21 & 14 & 10 & 9 & 9 & 11 & 10 & 6 & 4 \\
\hline $\mathrm{MM}^{\prime}$ & 17 & 18 & 18 & 12 & 12 & 8 & 6 & 4 & 6 & 4 & 3 & \\
\hline $\mathrm{MC}^{\prime}$ & 22 & 21 & 20 & 12 & 11 & 8 & 4 & 4 & 3 & 1 & & \\
\hline $\mathrm{RF}^{\prime}$ & 13 & 15 & 14 & 12 & 11 & 10 & 8 & 7 & 6 & 3 & 3 & 1 \\
\hline $\mathrm{RM}^{\prime}$ & 14 & 16 & 13 & 10 & 9 & 9 & 6 & 5 & 5 & 3 & 2 & 2 \\
\hline $\mathrm{RC}^{\prime}$ & 20 & 25 & 19 & 18 & 14 & 14 & 13 & 12 & 14 & 13 & 14 & 11 \\
\hline $\mathrm{CF}^{\prime}$ & 14 & 15 & 17 & 13 & 11 & 11 & 8 & 7 & 7 & 4 & 4 & 3 \\
\hline $\mathrm{CM}^{\prime}$ & 23 & 24 & 21 & 18 & 13 & 11 & 9 & 9 & 9 & 6 & 7 & 5 \\
\hline $\mathrm{CC}^{\prime}$ & 17 & 17 & 14 & 10 & 8 & 6 & 5 & 5 & 4 & 4 & 3 & 2 \\
\hline $\mathrm{SM}^{\prime}$ & 19 & 19 & 19 & 17 & 18 & 15 & 15 & 17 & 16 & 13 & 11 & 7 \\
\hline
\end{tabular}

- Par rapport à la fin de la $3^{\mathrm{e}}$ phase, il y a nettement plus de racines à tous niveaux pour toutes les modalités.

\subsection{Conclusions partielles.}

Un examen de l'évolution des caractéristiques du système radiculaire avec le temps permet d'établir les faits suivants :

- Toutes les racines qui se trouvent sous le niveau d'affleurement de la nappe pendant la phase de printemps meurent. L'enracinement vivant s'arrête à ce niveau.

- Le système radiculaire ne se développe que peu en longueur pendant la phase de printemps (tout au moins au cours de la $2^{\mathrm{e}}$ année); ce phénomène est évidemment logique pour les modalités à nappe, mais se vérifie également pour celles sans nappe; par contre sa densité augmente assez nettement, au moins pour certains traitements.

- L'enracinement se développe beaucoup, contrairement à ce qui se passe pour la pousse aérienne, pendant la phase d'été, tant en longueur qu'en densité; il dépasse alors très fortement le niveau auquel affleurait la nappe de printemps.

- La densité et surtout la profondeur de l'enracinement dépendent essentiellement du régime de printemps; à la fin de la phase de printemps évidemment, mais aussi à la fin de celle d'été, ce sont les 3 modalités $F$ (plus spécialement FF') dont l'enracinement est de loin le plus défavorable; il existe un seuil entre les nappes affleurant à $-7 \mathrm{et}-14 \mathrm{~cm}$; ce seuil est très net quel que soit le traitement d'été. 
- Par contre, les différences constatées pendant la phase de printemps entre les 3 autres traitements $(\mathrm{M}, \mathrm{R}$ et $\mathrm{C})$ n'apparaissent plus que fort atténuées à la fin de la phase d'été; les densités d'enracinement restent peu différentes les unes des autres, du moins globalement; quant aux longueurs, les différences entre les modalités $\mathrm{M}$ et les modalités $\mathrm{R}$ et $\mathrm{C}$ se sont fortement réduites dans leur ensemble; d'ailleurs la modalité $\mathrm{MF}^{\prime}$ atteint le fond du pot tout comme les modalités $\mathrm{C}$ et $\mathrm{R}$ (et sa densité d'enracinement est excellente).

- En raison du nombre plus réduit de pots (pour des causes matérielles) des 2 modalités annexes $\mathrm{SM}^{\prime}$ et $\mathbf{M H}^{\prime}$, nous n'avons pu examiner leur enracinement à la fin de toutes les phases. L'enracinement de SM' est très bon; il est en particulier pratiquement le plus dense à partir de $-12 \mathrm{~cm}$, au moins parmi les autres modalités $\mathbf{M}^{\prime}$; ce résultat est logique, la sécheresse de printemps devant favoriser la prospection du sol par les racines. Quant à celui de $\mathbf{M H}^{\prime}$, il s'arrête peu au-dessus de la nappe (à $-12 \mathrm{~cm}$ ); dans les zônes superficielles, sa densité est assez élevée, mais surtout son extension latérale est très grande, de loin la plus importante parmi toutes les modalités; ainsi cela se traduit à la fin de la $2^{\mathrm{e}}$ phase, malgré une profondeur d'enracinement parmi les plus faibles, par le poids sec de racines le plus élevé parmi toutes les modalités $\left(2,0 \mathrm{~g}\right.$ pour l'ensemble du pot $\mathrm{MH}^{\prime}$, alors que pour le pot arrivant en seconde position, qui est $\mathrm{SM}^{\prime}$, on trouve $1,8 \mathrm{~g}$ et pour le $3^{\mathrm{e}} 1,5 \mathrm{~g}$ ); les racines de toutes les modalités se développent donc beaucoup pendant l'été et, si elles ne peuvent le faire en longueur, elles se développent latéralement.

\section{3. - La mortalité}

Aucune mortalité ne s'est apparemment produite au cours de la $1^{\text {re }}$ phase.

A la fin de la $2^{\mathrm{e}}$ phase (été 1969) par contre, 27 plants sont morts, répartis ainsi : $\mathrm{FF}^{\prime}$ : $16, \mathrm{RF}^{\prime}: 5, \mathrm{FM}^{\prime}: 4, \mathrm{CM}^{\prime}$ et $\mathrm{FC}^{\prime}: 1$.

Au cours de la $3^{\mathrm{e}}$ phase, aucun plant ne meurt véritablement, mais un certain nombre donne des signes précurseurs à un dépérissement prochain (teinte jaunissante, pousse aérienne ne s'accroissant que très peu).

A la fin de la $4^{\text {e }}$ phase (été 1970), les plants morts depuis le début de l'expérience se répartissent de la manière suivante :

$\mathrm{FF}^{\prime}: 16, \mathrm{CF}^{\prime}: 14, \mathrm{RF}^{\prime}: 13, \mathrm{FM}^{\prime}: 4, \mathrm{FC}^{\prime}, \mathrm{RM}^{\prime}$ et $\mathrm{CM}^{\prime}: 1$ chacun.

(on ne peut comparer de façon absolue ces chiffres à ceux enregistrés à la fin de la $1^{\text {re }}$ phase, car certains pots ont été sacrifiés entre-temps).

C'est donc du régime d'été que dépend principalement la mortalité; des chiffres élevés n'ont été relevés que dans le cas d'une forte sécheresse d'été $\left(\mathrm{F}^{\prime}\right)$.

Cependant le régime de printemps intervient tout de même :

- Le traitement $\mathrm{F}$ est sans doute le plus défavorable des traitements de printemps, car d'une part c'est dans la seule modalité $\mathrm{FF}^{\prime}$ que l'on a enregistré un chiffre élevé de mortalité au cours de la $1^{\text {re }}$ année; et d'autre part, en dehors des 3 modalités $F^{\prime}$, ce n'est que pour FM' que l'on constate un chiffre de mortalité (4 individus) qu'on peut considérer peut-être comme n'étant pas provoqué par le hasard seulement.

- Parmi les modalités comportant une forte sécheresse d'été, seul MF' n'enregistre aucune mortalité, alors que pour toutes les autres le nombre de plants qui meurent est élevé. 


\section{4. - La nutrition}

Des analyses foliaires ont été effectuées à la fin de chaque phase.

La seule liaison entre traitement et composition foliaire qui ait pu être mise en évidence concerne l'azote.

A la fin de la $1^{\text {re }}$ phase, les teneurs des feuilles en azote, en $\%$ de poids, sont les suivantes :

$$
\mathrm{C}: 2,15-(\mathrm{S}: 1,76)-\mathrm{M}: 1,71-\mathrm{F}: 1,03
$$

La teneur en azote de F est donc nettement la plus faible de toutes.

A la fin de la $2^{\mathrm{e}}$ phase les 2 modalités pour lesquelles la teneur foliaire en azote est nettement la plus faible sont $\mathrm{RF}^{\prime}$ et $\mathrm{CF}^{\prime}$; mais rappelons que de nombreux plants de ces 2 modalités donnent au cours de la phase suivante des signes très nets de dépérissement : il n'est pas impossible qu'il y ait un rapport, et peut-être de cause à effet, entre les deux constatations. En dehors de ces 2 modalités, ce sont $\mathrm{FM}^{\prime}$ et $\mathrm{FF}^{\prime}$ qui ont la teneur foliaire en azote la moins élevée.

A la fin de la $3^{\mathrm{e}}$ phase on ne peut guère tirer d'enseignements de l'analyse foliaire; signalons seulement que la teneur la plus basse en azote est située dans les feuilles correspondant à la modalité $\mathrm{FC}^{\prime}: 1,45 \%$ alors qu'ensuite on trouve $\mathrm{CM}^{\prime}$ avec $1,63 \%$, puis $\mathrm{FM}^{\prime}$ avec $1,75 \%$.

A la fin de la $4^{\mathrm{e}}$ phase, les teneurs les plus basses en azote sont les suivantes :

$\mathrm{FF}^{\prime}: 1,77 \%-\mathrm{FM}^{\prime}: 2,08 \%-\mathrm{FC}^{\prime}: 2,14 \%-\mathrm{MM}^{\prime}$ et $\mathrm{RM}^{\prime}: 2,15 \%$; etc...

En résumé, il existe une liaison indéniable entre les modalités comportant un fort engorgement de printemps (nappe affleurant à $-7 \mathrm{~cm}$ ) et une teneur foliaire en azote faible par rapport aux autres traitements.

\section{IV. - SYNTHÈSE ET DISCUSSION}

Nous allons tenter d'effectuer une synthèse des différents résultats obtenus (croissance en hauteur, enracinement, mortalité, nutrition) tout en discutant de leur signification réelle et de leurs limites et en essayant si possible d'expliquer grâce à eux certains phénomènes que l'on observe dans les conditions naturelles.

\section{1. - Rythme de développement des plants}

La croissance en hauteur des parties aériennes se produit essentiellement pendant la phase de printemps; les tiges ne s'accroissent que très peu au cours de l'été.

Le rythme de développement du système radiculaire est très différent. Pendant la phase d'engorgement, toutes les portions de racines qui étaient situées préalablement sous le niveau d'affleurement de la nappe meurent. Pendant l'été, le système radiculaire se développe beaucoup, tant en longueur qu'en densité : il colonise à nouveau assez abondamment les zônes qui étaient engorgées au printemps.

Il est donc logique que, bien que les parties visibles (aériennes) d'un Épicéa n'évoluent 
guère pendant l'été, le régime hydrique du sol à cette saison influe sur la croissance en hauteur mais cette influence ne pourra se manifester qu'au cours du printemps suivant.

D'autre part, comme la présence d'une nappe au printemps n'empêche pas les racines de prospecter au cours de l'été les zônes qui étaient situées sous le niveau de la nappe, le comportement des plants dépend sans doute en partie des caractéristiques intrinsèques de ces zônes, en particulier des propriétés dont dépend la colonisation par les racines.

Ainsi par exemple l'enracinement se développera sans doute bien plus au cours de l'été dans un colluvium de bas de pente que dans un pseudogley sur substrat non remanié, pour un même type d'engorgement de printemps.

\section{2. - Influence des différents régimes de printemps sur le comportement des plants}

\subsection{Cas d'une nappe très superficielle $(-7 \mathrm{~cm})$.}

Le comportement des plants est très différent de ce qu'il est lorsque la nappe est un peu plus profonde $(-14 \mathrm{~cm})$ : il semble exister un seuil entre ces 2 niveaux.

Ce seuil se manifeste plus ou moins nettement suivant la caractéristique considérée. En ce qui concerne la pousse aérienne, pour un même traitement d'été, c'est la nappe à $-7 \mathrm{~cm}$ qui constitue nettement le traitement de printemps le plus défavorable. Mais, en valeur absolue, un bon approvisionnement en eau pendant l'été $\left(\mathrm{C}^{\prime}\right)$ compense en grande partie l'effet défavorable d'une nappe de printemps à $-7 \mathrm{~cm}$ : si les modalités $\mathrm{FF}^{\prime}$ et $\mathrm{FM}^{\prime}$ sont défavorables, il n'en est absolument pas de même pour $\mathrm{FC}^{\prime}$.

Pour l'enracinement, qu'il soit de printemps ou d'été, le seuil se manifeste de façon encore bien plus nette que pour la croissance en hauteur : en effet dans ce cas les trois modalités $\mathrm{F}$ sont les plus défavorables de toutes, quel que soit le traitement d'été.

Cette différence entre le comportement des parties aériennes et souterraines pour la modalité à fort engorgement de printemps mais bon approvisionnement en eau pendant l'été $\left(F C^{\prime}\right)$ est logique et correspond à ce que l'on observe dans les conditions naturelles; ainsi, les épicéas situés dans des bas-fonds ont souvent une excellente croissance même si la nappe de printemps est superficielle : ces épicéas se trouvant pendant l'été dans des conditions très favorables pour l'approvisionnement en eau, un développement important du système radiculaire est inutile pour que l'alimentation hydrique soit correcte.

Évidemment cette comparaison des résultats expérimentaux avec les phénomènes observés dans la nature ne concerne directement que les jeunes plantations; cependant il faut signaler une certaine analogie entre ces résultats et le comportement des peuplements adultes d'épicéas ; il s'agit peut-être d'une caractéristique de l'espèce qui se manifeste pendant toute la vie de l'arbre, bien que d'une manière variable suivant l'âge.

C'est donc bien l'alimentation en eau pendant l'été qui constitue le facteur critique essentiel de la croissance pour de nombreuses plantations d'Epicéa sur pseudogley; ce facteur dépend d'une part des conditions topographiques, d'autre part du développement du système radiculaire (dont est fonction l'utilisation des réserves hydriques du sol); or ce dernier dépend du niveau d'affleurement de la nappe au cours du printemps, une nappe très superficielle (par exemple affleurant à $-7 \mathrm{~cm}$ ) constituant l'éventualité la plus défavorable.

Le résultat des analyses foliaires a montré que ce seuil (situé entre les nappes affleurant 
à -7 et $-14 \mathrm{~cm}$ ) se manifestait également sur la nutrition azotée des plants. La recherche d'une explication pour ce phénomène peut donner lieu à diverses hypothèses (développement insuffisant des racines, nappe perturbant la minéralisation de l'humus, myccorhizes tuées, racines en mauvais état sanitaire, etc...)

Enfin en ce qui concerne la mortalité, ce seuil ne constitue pas son facteur déterminant. Il se manifeste cependant, en particulier par le phénomène suivant : parmi les plants qui subissent une forte sécheresse du sol pendant l'été (seul traitement provoquant un taux élevé de mortalité), on n'enregistre une mortalité pendant la première année que pour ceux dont l'engorgement de printemps était très accentué.

\subsection{Autres traitements de printemps.}

Une nappe affleurant à une profondeur intermédiaire $(-14 \mathrm{~cm}$, traitement $\mathrm{M})$ correspond, quel que soit le traitement d'été, à une croissance en hauteur parmi les meilleures; s'il y a sécheresse d'été (moyenne ou forte), ce traitement $M$ donne des résultats supérieurs à tous les autres.

D'autre part, $\mathrm{MF}^{\prime}$ est la seule des modalités à forte sécheresse d'été à ne subir aucune mortalité.

L'enracinement d'été des modalités $\mathrm{M}$, s'il est parfois un peu moins profond que pour $\mathrm{R}$ ou $\mathrm{C}$, est au moins aussi dense; en particulier $\mathrm{MF}^{\prime}$ a très nettement l'enracinement le plus dense de toutes les modalités subissant une forte sécheresse d'été et ses racines atteignent une même longueur que $\mathrm{RF}^{\prime}$ et $\mathrm{CF}^{\prime}$ : ces faits sont à rapprocher de ceux signalés ci-dessus au sujet de la mortalité.

La nappe à - $14 \mathrm{~cm}(\mathrm{M})$ constitue donc le traitement de printemps le plus favorable aux jeunes plants d'Épicéa. Ensuite, au moins pour la croissance en hauteur, c'est la nappe à $-21 \mathrm{~cm}(\mathrm{R})$; ces 2 traitements sont préférables de ce point de vue à $\mathrm{C}$, c'est-à-dire à un approvisionnement convenable en eau mais en l'absence de nappe : c'est la présence d'eau libre, c'est-à-dire un potentiel capillaire pratiquement nul, à proximité immédiate de nombreuses racines qui constitue le régime le plus favorable aux plants d'Epicea (non seulement au printemps, mais également l'été : la supériorité de la modalité $\mathrm{MH}^{\prime}$ par rapport à $\mathrm{MC}^{\prime}$ le montre). Pour de tous jeunes plants, cette nappe doit être assez superficielle : M $(-14 \mathrm{~cm})$ est préférable à $\mathrm{R}(-21 \mathrm{~cm})$ pour la croissance et surtout la survie; mais il est assez probable que certains des niveaux considérés, en particulier le seuil (situé entre -7 et $-14 \mathrm{~cm}$ ) et l'optimum ( $-14 \mathrm{~cm}$ ) se décalent vers le bas au fur et à mesure du développement des plants.

Terminons cette étude des nappes par une remarque d'ordre général : lorsqu'il y a nappe, l'enracinement se développe abondamment jusqu'à proximité immédiate de la surface d'eau libre (entre le niveau même de l'eau et $2 \mathrm{~cm}$ au-dessus) : même la partie inférieure de la frange capillaire est favorable au développement des racines d'Épicéa, la forte teneur du sol en eau ne les tue pas.

La modalité SM' est évidemment très éloignée des conditions d'un pseudogley. Remarquons seulement que la croissance en hauteur qui lui correspond est assez bonne et l'enracinement profond et dense : le fort enracinement a sans doute compensé en partie la pénurie relative en eau pendant le printemps. Cela confirme, si cela était nécessaire, que l'Épicéa est une essence fort plastique vis-à-vis des conditions écologiques. 


\section{3. - Influence des différents régimes d'été sur le comportement des plants}

\subsection{Cas d'un approvisionnement convenable en eau pendant l'été (traitement $C^{\prime}$ )}

A ce traitement correspond toujours une excellente survie ainsi qu'une bonne croissance en hauteur des plants quelque soit le traitement de printemps, croissance toujours supérieure à celle des modalités à sécheresse d'été ( $\mathbf{M}^{\prime}$ ou $\mathrm{F}^{\prime}$ ), à traitement de printemps équivalent. Par contre l'enracinement est influencé principalement par le traitement de printemps.

On peut donc supposer, et c'est logique, qu'une pluviosité assez abondante pendant l'été favorise plus qu'un été sec la croissance en hauteur de plants d'Épicéa sur pseudogley lors du printemps suivant.

\subsection{Cas d'une forte sécheresse d'été (traitement $F^{\prime}$ )}

En ce qui concerne la croissance en hauteur et l'enracinement, ce traitement ne semble pas plus défavorable (au contraire même parfois pour l'enracinement) qu'une sécheresse moins accentuée $\left(\mathrm{M}^{\prime}\right)$.

Par contre c'est de très loin le traitement le plus défavorable pour la survie des plants (nous avons indiqué qu'il se traduisait par une forte mortalité, sauf dans le cas d'une nappe de printemps à $-14 \mathrm{~cm}$ ).

C'est pourquoi il faut considérer avec prudence le résultat selon lequel une forte sécheresse ne serait pas plus défavorable à la croissance en hauteur de plants d'Épicea qu'une sécheresse moins prononcée. En effet nous avons pu constater que les plants qui devaient mourir au cours de la $4^{\mathrm{e}}$ phase n'avaient qu'une très faible croissance pendant la phase précédente (printemps 1970), mais évidemment leur pousse n'a pas été prise en considération lors du calcul des moyennes. Dans les conditions de terrain, la sécheresse d'été, si elle est très souvent insuffisante pour tuer les arbres, se traduit peut-être seulement par une diminution de leur croissance.

\subsection{Cas d'une nappe permanente (traitement $H^{\prime}$ )}

Nous sortons évidemment du cadre des pseudogleys. Néanmoins on peut peut-être assimiler partiellement cette modalité aux conditions rencontrées dans d'autres types de sols par exemple les gleys, bien que la nappe subisse alors des fluctuations saisonnières, ou bien les amphi-gleys de bas-fonds.

La pousse aérienne est dans ce cas nettement la plus forte de toutes; nous avons indiqué précédemment ce qu'on pouvait en penser; quant aux racines, si elles ne se développent jamais bien profondément en raison de la barrière constituée par la nappe, elles prennent une extension latérale très forte. C'est pourquoi, dans des conditions naturelles qui se rapprocheraient de celles de cette modalité, il serait utile de prévoir une densité de peuplement assez faible, afin de réduire au maximum la forte concurrence entre racines.

\section{4. - Remarques}

Il n'est pas interdit de penser que, dans les conditions naturelles, la présence d'une nappe temporaire pendant de nombreuses années successives provoque la formation dans le sol 
de corps toxiques pour les arbres (par exemple ions aluminium ou manganèse) : alors l'effet de la nappe serait bien plus défavorable pour le comportement de l'Épicéa que ce que l'on a pu constater dans nos pots au cours d'une expérience qui n'a duré que 2 ans.

En fait la bonne croissance de nombreuses plantations d'Épicéa sur pseudogley semble montrer qu'il n'en est rien.

\section{V. - CONCLUSIONS}

Ce travail, destiné à étudier l'influence, sur le comportement de très jeunes plants d'Épicéa, de différents traitements de printemps, en particulier plusieurs niveaux de nappe (un même niveau restant constant pendant toute la période de printemps) suivis de diverses intensités de dessèchement du sol au cours de l'été, a fourni les principaux résultats suivants :

- Le traitement comportant la présence d'une nappe très superficielle (affleurement à $-7 \mathrm{~cm}$ ) est le plus défavorable de tous les traitements de printemps.

- d'une part pour la croissance en hauteur des plants; néanmoins celle-ci est relativement bonne par rapport à l'ensemble de 12 modalités complètes si l'approvisionnement en eau pendant l'été est convenable, ce qui montre que le facteur défavorable à la croissance des plants d'Épicéa sur sols à pseudogley est constitué par la présence d'une nappe très superficielle au printemps, mais à condition qu'elle soit suivie d'un dessèchement du sol pendant l'été,

- d'autre part pour le développement du système radiculaire; en effet d'une manière générale quel que soit le niveau de la nappe, toutes les racines qui se trouvent engorgées périssent, mais elles se développent à nouveau abondamment au cours de l'été suivant; or, dans le cas d'une nappe, très superficielle le système radiculaire demeure le moins dense et le moins profond de tous, quel que soit le traitement d'été.

- également pour la survie des plants; de ce point de vue, ce n'est que parmi les plants subissant un très fort dessèchement du sol pendant l'été que l'on enregistre un taux élevé de mortalité; or, parmi ces plants, seuls ceux pour lesquels l'engorgement était très superficiel meurent au cours de la $1^{\text {re }}$ année de repiquage.

- et enfin pour la nutrition azotée : il existe une liaison très nette entre une faible teneur foliaire en azote et une nappe de printemps très superficielle.

- Un engorgement un peu moins superficiel (nappe affleurant à $-14 \mathrm{~cm}$ ) constitue par contre l'éventualité la plus favorable,

- d'une part pour la croissance en hauteur des plants,

- et surtout pour leur survie : de toutes les modalités comportant un très fort dessèchement du sol au cours de l'été, seule celle subissant ce traitement de printemps (nappe à $-14 \mathrm{~cm}$ ) échappe à un taux de mortalité élevé; il est possible que cela soit en relation avec l'important développement du système radiculaire de ces plants.

- L'engorgement que l'on vient de considérer est préférable à un engorgement plus profond (nappe à $-21 \mathrm{~cm}$ ); mais tous deux sont plus efficaces, pour la croissance en hauteur, qu'un approvisionnement convenable en eau mais sans nappe : un potentiel capillaire pra- 
tiquement nul à proximité immédiate des racines constitue, pour les plants d'Épicéa, l'éventualité la plus favorable (à condition que la nappe ne soit pas excessivement superficielle).

Cette expérience n'était évidemment destinée à aborder qu'un aspect du problème. Aussi serait-il utile de la compléter par d'autres études. Ainsi, s'il est établi qu'un engorgement très superficiel perturbe fortement le développement des parties aériennes et souterraines des plants d'Épicéa, il reste à déterminer les durées et périodicités critiques de l'engorgement superficiel et même de la submersion (eau affleurant au niveau du sol).

Par ailleurs on pourra tenter de tester l'effet de certaines interventions possibles (fertilisation par exemple) dans la lutte contre un engorgement excessif.

Enfin, ces mêmes études pourraient s'avérer instructives dans le cas d'autres essences (tel le Douglas) plus sensibles à la présence d'un excès d'eau dans le sol.

Reçu pour publication en juillet 1971.

\section{SUMMARY}

INFLUENCE OF SOIL WATER LOGGING DURING SPRING, FOLLOWED BY

SUMMER DRYNESS, ON THE BEHAVIOUR OF YOUNG PLANTS OF NORWAY SPRUCE

Soils with temporary hydromorphy (soil with pseudogley) cover important surfaces in France, particularly in the North-East of the country. This soil water logging brings on some conditions unfavourable to forest stands, that at all stages of trees life. The purpose of this experiment is to explain partly the behaviour of young forest plants on this kind of soil.

\section{Methods}

Norway Spruce was chosen because it is the tree species the most used, until now, for this type of soil in France.

Seedlings 1-0 were transplanted in large earthen pots $(30 \mathrm{~cm}$ high and $30 \mathrm{~cm}$ of internal diameter), at the rate of 7 seedlings a pot. The soil of the pots was composed by the horizons $A_{1}$ and $A_{9} g$ of a soil with pseudogley (table I). The pots were placed into large metallic buckets into which was set a water-table. The experiment was placed into a greenhouse.

Each pot is subjected to two consecutive treatments : one during the end of winter and the spring, the other during summer. There is 4 spring treatments : $\mathrm{C}=$ soil maintained about at field capacity, $\mathrm{F}, \mathrm{M}$ and $\mathrm{R}=$ level of water-table at respectively $-7,-14$ and $-21 \mathrm{~cm}$. The tree summer treatments are : $\mathrm{C}^{\prime}=$ soil maintained about at field capacity, $\mathrm{F}^{\prime}=$ severe dryness (soil moistened only about one time a month), $\mathbf{M}^{\prime}=$ mean dryness (soil moistened with a double periodicity). By associating two by two spring treatments and summer treatments, 12 complete treatments are obtained.

The measures concern height growth of the plant, their rooting (length and dentsity), the mortality; also foliar analysis are intended to control the nutrition level.

\section{Results}

Here are the main results we have obtained :

1 - The treatment with a very shallow water-table (F) is the most unfavourable among the four spring treatments :

- at one side for the height growth of the plants (tables 2 and 3); nevertheless this growth is relatively good compared to the whole 12 treatments, if the supply of water is suitable during the summer (treatment $\mathrm{FC}^{\prime}$ ); this shows us that the factor unfavourable to the growth of Spruce's young plants on soil with pseudogley is formed by the presence of a very shallow water-table during spring, but on condition that it is followed by a dryness of the soil during summer;

- at the other side for the development of rooting system (tables 4 to 10 ); indeed, generally, whatever the level of water-table is, all water logged roots die but they develop again plentifully during the next summer; now, in the case of a very shallow water-table $(F)$, the rooting system remains the less dense and the less deep, whatever the summer treatment is; 
- also for the plants survival. It is only among the plants undergoing a very severe dryness of the soil during summer $\left(\mathrm{F}^{\prime}\right)$ that it is a high rate of mortality; now, among these plants, only those for which water logging was very shallow $\left(\mathrm{FF}^{\prime}\right)$ die during the first year after transplanting;

- and, at last, for nutrition in nitrogen : there is a strong relation beetween a low rate of nitrogen in the needles and a very shallow spring water-table $(\mathrm{F})$.

2 - A water-logging a little less shallow (treatment $M$ ) is, on the other hand, the most favourable occurence :

- for the growth of the plants; whatever the summer treatment is (dryness or no dryness), these plants have a very good height growth;

- and for their survival : among all treatments with a severe soil dryness during summer $\left(\mathrm{F}^{\prime}\right)$, only that undergoing this spring treatment $\left(\mathrm{MF}^{\prime}\right)$ has not a high rate of mortality; perhaps that is in relation with the important development of the rooting system of these plants.

3 - The preceding water-logging $(M)$ is preferable to a more deep one (R); but both are more efficient for the height growth than a satisfactory supply but without water-table (C) : an almost null capillary potential by the roots is, for young Spruce's plants, the most favourable occurence (provided that water-table is not excessively shallow).

\section{ZUSAMMENFASSUNG}

DER EINFLUSS DER FRUHJAHRS-STAUNASSE UND DER SOMMERTROCKENHEIT

AUF DES VERHALTEN VON JUNGEN FICHTENPFLANZEN

Der Versuch wurde an Fichtensämlingen (Picea abies L.), welche in $30 \mathrm{~cm}$ hohen Tontöpfen verschult, waren, durchgeführt und zwei Jahre lang im Glashaus beobachtet. Zwölf verschiedene Behandlungen wurden untersucht :

Frühjahrsbehandlungen :

- C : Die Bodenfeuchte entspricht der Feldkapazität,

$-\mathrm{F}, \mathrm{M}, \mathrm{R}$ : Der Wasserspiegel wird auf $-7,-14$ und $-21 \mathrm{~cm}$ gehalten.

Sommerbehandlungen :

- $\mathrm{F}^{\prime}$ : starke Trockenheit; es wird ca. einmal pro Manot Wasser zugesetzt,

- $\mathrm{M}^{\prime}$ : mittlere Trockenheit; es wird ca. zweimal pro Monat Wasser zugesetzt,

$-\mathrm{C}^{\prime}$ : Die Bodenfeuchte entspricht der Feldkapazität.

Es zeigten sich im wesentlichen die folgenden Ergebnisse :

- Die Behandlung F (Wasserspiegel nahe an der Oberfläche) zeigte das geringste Höhenwastum; dies ist jedoch nicht der Fall, wenn im Sommer eine gute Wasserversorgung (Behandlung $C^{\prime}$ ) erfolgt. Die Behandlung $\mathrm{F}$ bewirkt gleichfalls, unabhängig von der Sommerbehandlung, die geringste Wurzeldichte und Wurzeltiefe, sowie eine sehr schlechte Stickstoffernährung der Pflanzen.

- Die Behandlung M (mittlerer Wasserspiegel) zeigt bezüglich Höhenwaschstum und Ausfall die besten Ergebnisse.

- Die Behandlungen M und R (Wasserspiegel in mittlerer und grösserer Tiefe) haben einen günstigeren Einfluss auf das Höhenwaschstum, als eine gute Wasserversorgung ohne Grundwasserspiegel (Behandlung C).

- Die Sommerbehandlung F' (starke Trockenheit) zeigt als Eingzige ein hohes Ausfallprozent, mit Ausnahme der Frühjahrsbehandlung M.

\section{RÉFÉRENCES BIBLIOGRAPHIQUES}

Kreutzer K., 1961 - «Wurzelbildung junger Waldbaüme auf Pseudogley-böden » Forstw. Centbl., 80 $(11-12), 356-392$.

Lévy G., 1969 - Importance des propriétés du sol pour l'enracinement de Picea excelsa et de Pinus silvestris. Ann. sci. for., 1968, 25 (3), 157-188.

Leyton L., Rousseau L. Z., 1968 - Root growth of tree seedlings in relation to aeration. In «the Physiology of forest trees $\gg$, K. V., Thimann, Ronald Press, New-York, 23, 467-475.

PÉrigaud S., 1967 - Effet du manque d'oxygène et de l'excès d'eau au niveau des racines sur la croissance et la nutrition globale de la plante. Ann. Agron., 18 (5), 485-506. 